

\title{
Transient modal radiation of axisymmetric sources: application to loudspeakers.
}

Nicolas Quaegebeur, Antoine Chaigne, Guy Lemarquand

\section{To cite this version:}

Nicolas Quaegebeur, Antoine Chaigne, Guy Lemarquand. Transient modal radiation of axisymmetric sources: application to loudspeakers.. Journal of Applied Acoustics, 2010, 71 (4), pp.335-350. 10.1016/j.apacoust.2009.10.003 . hal-00462370

\section{HAL Id: hal-00462370 \\ https://hal.science/hal-00462370}

Submitted on 17 Mar 2010

HAL is a multi-disciplinary open access archive for the deposit and dissemination of scientific research documents, whether they are published or not. The documents may come from teaching and research institutions in France or abroad, or from public or private research centers.
L'archive ouverte pluridisciplinaire HAL, est destinée au dépôt et à la diffusion de documents scientifiques de niveau recherche, publiés ou non, émanant des établissements d'enseignement et de recherche français ou étrangers, des laboratoires publics ou privés. 


\title{
Transient modal radiation of axisymmetric sources: application to loudspeakers.
}

\author{
Nicolas Quaegebeur*, Antoine Chaigne \\ UME-ENSTA, Chemin de la Hunière, 91761 Palaiseau Cedex, France
}

Guy Lemarquand

LAUM, UMR CNRS 6613, Avenue O. Messiaen, 72085 Le Mans Cedex 9, France

\begin{abstract}
For high amplitudes of vibrations, loudspeakers are subject to nonlinear phenomena that are responsible for audible distortions. In order to describe the complex dynamics of the system, the displacement field as well as the radiated sound pressure must be expressed in the time-domain. Thus the present study proposes a transient model of the acoustic radiation of axisymmetric structures. The pressure field is approximated by the Rayleigh integral corresponding to a monopole source distribution over the non-planar vibrating surface. The displacement field is expanded on the linear modes of the structure and a change of variables in the Rayleigh integral is then proposed in the case of a monotonic profile function to compute the Spatial Impulse Response associated to each mode of vibration efficiently. The results are compared to the formulation obtained in the case of planar and spherical sources. The method of calculation is then derived in the case of a typical loudspeaker profile (association of a truncated cone with a spherical cap). Finally, the present approach is used to estimate the nonlinear radiation pattern of a prototype loudspeaker and predictions are compared to measurements in anechoic room.
\end{abstract}

Key words: loudspeaker, nonlinear vibrations, transient, radiation, Rayleigh PACS: 43.20.Px, 43.20.Tb, 43.20.Rz, 43.40.-r, 43.40.Rj

\footnotetext{
* Corresponding author.

Email addresses: nicolas.quaegebeur@gmail.com (Nicolas Quaegebeur), antoine.chaigne@ensta.fr (Antoine Chaigne), guy.lemarquand@univ-lemans.fr (Guy Lemarquand).
} 


\begin{tabular}{|llll|}
\hline Nomenclature & & \\
$a$ & external radius & $\mathbf{x}_{\mathbf{i}}$ & impact point \\
$c$ & celerity of sound in air & $\theta_{i}$ & angle of the impact point \\
$h_{0}(r)$ & profile function & $\Phi_{p}$ & $p_{\text {th }}$ natural mode for the \\
$P(x, t)$ & Acoustic pressure & & flexural displacement \\
$q_{p}$ & time-function associated & $\rho$ & density of air \\
& to the $p_{\text {th }}$ natural mode & $\sigma$ & ratio of densities \\
$r$ & radial coordinate & $\mathcal{B}_{c}=\left(\overrightarrow{u_{x}}, \overrightarrow{u_{y}}, \overrightarrow{u_{z}}\right)$ & Cartesian basis \\
$R$ & curvature radius & $\mathcal{B}_{i}=\left(\overrightarrow{t_{i x}}, \overrightarrow{t_{i y}}, \overrightarrow{n_{i}}\right)$ & local basis \\
& for the spherical cap & $(x, y, z)$ & coordinates in the basis $\mathcal{B}_{c}$ \\
$S$ & axisymmetric structure & $\left(r_{2}, \phi\right)$ & coordinates in the basis $\mathcal{B}_{i}$ \\
$t$ & time variable & $\overrightarrow{T_{\alpha}}$ & tangent vector with respect \\
$t^{\prime}$ & integration time & & to the variable $\alpha$ \\
$\mathbf{x}$ & observation point & $\mathcal{H}_{p}$ & Spatial Impulse Response \\
$\mathbf{x}_{\mathbf{S}}$ & integration point & & associated to mode $p$ \\
\hline
\end{tabular}

\section{INTRODUCTION}

The aim of an electrodynamic loudspeaker is to transform an electrical signal into sound. Such a transduction is expected to be linear. However, for high levels of vibrations, nonlinear phenomena appear and are responsible for audible distortions. The various sources of nonlinearities can be separated into two parts [1,2]: "electrical" nonlinearities due to the large displacement of the coil in the permanent magnet, and "mechanical" nonlinearities due to large displacements of the moving parts of the system (geometrical nonlinearities). In order to describe the complex dynamics of the system subjected to large amplitude motion, the displacement field must be expressed in the timedomain. In that case, a common way to compute the transient displacement field is to expand the transverse displacement of the structure onto the linear modes. This method has received much attention in the literature in the case of circular sources [3], axisymmetric sources [4] and recently in the case of loudspeaker like-structure [5] but none of the cited studies include the acoustic radiation of the structure. In order to compute the acoustic field radiated by thin structures subjected to large amplitude motion, the present article 
proposes a transient model of acoustic radiation that takes advantage of the modal expansion.

Transient acoustic radiation of planar sources vibrating in an infinite planar baffle has received much attention in the literature. The Rayleigh surface integral is in that case an exact expression for predicting the acoustic pressure produced by such sources at any observation point. An extensive review of the various approaches which have been used to evaluate this radiation integral has been given by Harris[6]. The most common way to compute the transient radiation from such sources is to evaluate the so-called Spatial Impulse Response (S.I.R.) as described by Stepanishen [7]. This formulation has been developed initially in the case of an uniform velocity for a circular source, and has then been extended to handle the case of nonuniform velocity patterns $[8,9]$ using a modal expansion of the displacement field.

For nonplanar sources, the Rayleigh surface integral is no longer valid, since diffraction effects appear. An analytical formulation of the transient radiation of a body of arbitrary shape has been derived by $\mathrm{Hu}$ and $\mathrm{Wu}[10,11]$, who expressed the acoustic pressure as a sum of integrations of simple and doublet source distributions and their couplings induced by the normal and tangential components of the particle velocity. Under that consideration, the Rayleigh integral represents the first term of this decomposition. This approximation is commonly used to compute transient radiation of spherical radiators [12-16], but the range of validity (in terms of geometrical configuration and wavenumber) is rarely specified.

Only the case of spherical sources has been treated and only few studies concern the acoustic radiation of axisymmetric sources. Farn [17] proposed a numerical method based on source-density method (approximation of the radiating surface by triangular surface elements) and Guyomar [18] used series expansion of the source velocity spatial distribution. More recently, Stepanishen [19] adapted a mean-square error method to predict harmonic radiation of bodies of arbitrary shape but the radiation of transients remains a problem. Other numerical approaches using retarded potentials combined to boundary elements in the time domain have can also be found in the literature $[20,21]$ and give appropriate results but calculation costs remain a problem.

In the present study, a time-domain formulation is adopted to predict the modal sound radiation from axisymmetric sources with a nonuniform vibratory distribution. The pressure field is approximated by the Rayleigh integral corresponding to a monopole source distribution over the nonplanar vibrating surface. The diffraction effects are neglected in the present approach and let for future work. The displacement field is expanded onto the linear modes of the structure and a change of variables in the Rayleigh integral is proposed in the case of a monotonic profile function to compute the Spatial Impulse Response associated to each mode of vibration efficiently. The results are compared to the exact formulation obtained in the case of planar sources [6] and to numerical results obtained by Suzuki and Tichy $[22,23]$ with the spherical harmonics method in the case of spherical radiators. The method of calculation is then 
derived in the case of a typical loudspeaker profile (association of a truncated cone with a spherical cap). Finally, the present approach is used to estimate the nonlinear radiation pattern of a prototype loudspeaker and predictions are compared to measurements in anechoic room.

\section{TIME-DOMAIN RADIATION}

\subsection{Geometry of the problem}

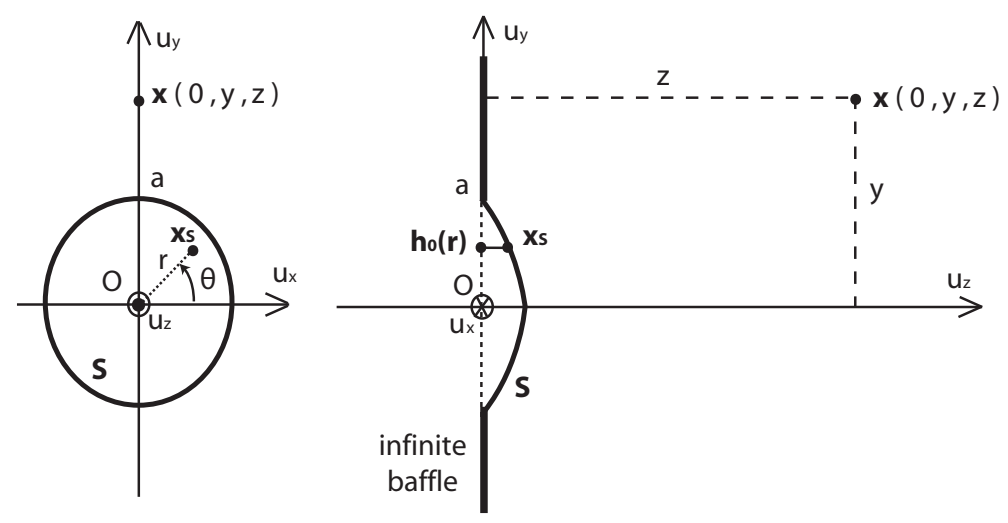

Fig. 1. Geometry of the considered structure. The points $\mathbf{x}$ and $\mathbf{x}_{\mathbf{s}}$ represent the receiver and an integration point on the structure respectively. The profile function is denoted by $h_{0}(r)$ and corresponds to the projection in the plane $z=0$ of the $u_{z}$ coordinate of the point $\mathbf{x}_{\mathbf{s}}$.

In the present study, attention is paid to the acoustic radiation of a baffled axisymmetric body $S$ as represented in Fig. 1. Let $a$ denote the external radius, $h_{0}(r)$ the axisymmetric profile function of the considered structure and $h_{\max }$ its depth (defined as the maximum of the profile function). The profile function only depends on the radial coordinate $r$ since $S$ is assumed axisymmetric. For the same reason, the observation point $\mathbf{x}$ depends on two coordinates $(y, z)$ in the Cartesian space only. The profile function $h_{0}(r)$ is called monotonic if its derivative $\dot{h_{0}}(r)$ has a constant sign and called non-monotonic otherwise. A concave structure refers to a negative profile function $h_{0}(r)<0$ while a convex structure relates to a positive profile function. In the following equations, $\mathbf{x}_{\mathbf{S}}$ denotes a point of the structure with cylindrical coordinates $\left(r, \theta, h_{0}(r)\right)$.

\subsection{Nonlinear vibration of loudspeaker-like structures}

Previous studies $[2,5]$ mention that the nonlinear vibrations of loudspeaker-like structure can be modeled using a nonlinear lumped parameters model coupled 
with a nonlinear model of the diaphragm vibrations. This formulation allows to include typical electrical nonlinearities that appear in the low frequencies domain but also geometrical nonlinearities that appear around mechanical resonances of the structure. The approach is based on an axisymmetric modal decomposition of the displacement field of the structure:

$$
w(r, t)=\sum_{p=0}^{\infty} \Phi_{p}(r) q_{p}(t) .
$$

where the functions $\Phi_{p}(r)$ in Eq. (1) represent the modal shapes (that can be either measured or calculated) and the functions $q_{p}(t)$ represent the time function associated to mode $p$. Using a state-space formulation of the nonlinear electromechanical problem[2], one obtains:

$$
\dot{Y}=\mathbf{A} Y+B(Y) u(t)+N L_{e}(Y)+N L_{g}(Y)
$$

where $Y$ is the state vector (time functions $q_{p}(t)$ and their first derivative $\left.\dot{q}_{p}(t)\right)$, $A$ denotes the linear dynamics of the problem, $B$ the excitation vector, $u(t)$ the input signal, $N L_{e}(Y)$ and $N L_{g}(Y)$ contain respectively all the electrical, mechanical and geometrical nonlinear terms of the electromechanical transduction. Eq. (2) is solved numerically using the fixed point method and Runge-Kutta algorithms, so that the global displacement field is obtained from the calculation of the state vector $Y$ using modal reconstruction Eq. (1). This global nonlinear formulation describing the complex dynamics of a typical loudspeakers needs to be directly solved in the time-domain. In order to compute the associated radiated sound pressure field for high amplitudes of vibration, a time-domain formulation of sound radiation from axisymmetric sources with a nonuniform vibratory distribution has to be adopted, as presented below.

\subsection{Integral formulation}

The sound pressure field $P(\mathbf{x}, t)$ is solution of the well known KirchhoffHelmholtz integral [24], that can be reduced to the Rayleigh's integral when considering weakly curved structures, i.e. for $h_{0}(r) \ll a$ :

$$
P(\mathbf{x}, t)=\rho \int_{0}^{t} \iint_{S} G\left(\mathbf{x}, \mathbf{x}_{\mathbf{S}}, t, t^{\prime}\right) \frac{\partial V_{n}\left(\mathbf{x}_{\mathbf{S}}, t^{\prime}\right)}{\partial t^{\prime}} d \mathbf{x}_{\mathbf{S}} d t^{\prime}
$$

Using the axisymmetric modal decomposition Eq. (1), the Rayleigh's integral is directly computed in the time-domain by separating the space and the time integration using the Spatial Impulse Response principle[19]:

$$
P(\mathbf{x}, t)=\rho \int_{0}^{t} \sum_{p=0}^{\infty} \mathcal{H}_{p}\left(\mathbf{x}, t, t^{\prime}\right) \ddot{q}_{p}\left(t^{\prime}\right) d t^{\prime}
$$


where the Spatial Impulse Response (S.I.R.) $\mathcal{H}_{p}$ associated to mode $p$ is introduced as follow:

$$
\mathcal{H}_{p}\left(\mathbf{x}, t, t^{\prime}\right)=\iint_{S} G\left(\mathbf{x}, \mathbf{x}_{\mathbf{S}}, t, t^{\prime}\right) \Phi_{p}\left(\mathbf{x}_{\mathbf{S}}\right) \overrightarrow{u_{z}} \cdot \overrightarrow{d \mathbf{x}_{\mathbf{S}}}
$$

where $\overrightarrow{u_{z}} \cdot \overrightarrow{d \mathbf{x}_{\mathbf{S}}}$ denotes the dot product between these 2 vectors. The analytical formulation of those functions can be obtained through an appropriate change of coordinates in the case of planar [19] or spherical structures [13]. However, in the general case, the direct calculation in the time-domain is not obvious due to the presence of the Dirac delta function. The following section proposes an appropriate change of variable in order to compute directly the S.I.R. functions for an axisymmetric source.

\section{CALCULATION OF THE RAYLEIGH INTEGRAL IN THE TIME-DOMAIN}

\subsection{Geometrical considerations}

The aim of the following section is to simplify the calculation of the impulse response defined in Eq. (5). Geometrical considerations are firstly made in order to separate 3 areas of calculations where the properties of the sound pressure field differ. Then, a new local basis is proposed using those geometrical considerations and the S.I.R. are simply expressed using the new set of coordinates. This method, developped in the case of planar and spherical sources is extended in the present study to the case of axisymmetric sources and offers the advantage of suppressing the Dirac delta function in Eq. (5) (using the convolution theorem), so that the final formulation is reduced to a simple integration.

The Spatial Impulse Response $\mathcal{H}_{p}$ in Eq. (5) has a compact support $\left[t_{i} ; t_{o}\right]$ where $t_{i}$ denotes the propagation time between the receiver $\mathbf{x}$ and the nearest point of the structure $\mathbf{x}_{\mathbf{i}}$ and $t_{o}$ represents the propagation time between the receiver and the farthest point of the structure $\mathbf{x}_{\mathbf{o}}$. The points $\mathbf{x}_{\mathbf{i}}=\left(0, r_{i}\right)$ and $\mathbf{x}_{\mathbf{o}}=\left(0, r_{o}\right)$ are solutions of:

$$
\begin{aligned}
& \left|\mathbf{x}-\mathbf{x}_{\mathbf{i}}\right|=\min _{(r, \theta)}\left(\left|\mathbf{x}-\mathbf{x}_{\mathbf{S}}\right|\right), \\
& \left|\mathbf{x}-\mathbf{x}_{\mathbf{o}}\right|=\max _{(r, \theta)}\left(\left|\mathbf{x}-\mathbf{x}_{\mathbf{S}}\right|\right) .
\end{aligned}
$$

Let $\mathbf{x}_{\mathbf{h}}$ and $\mathbf{x}_{\mathbf{b}}$ denote the points located respectively at the top and at the 


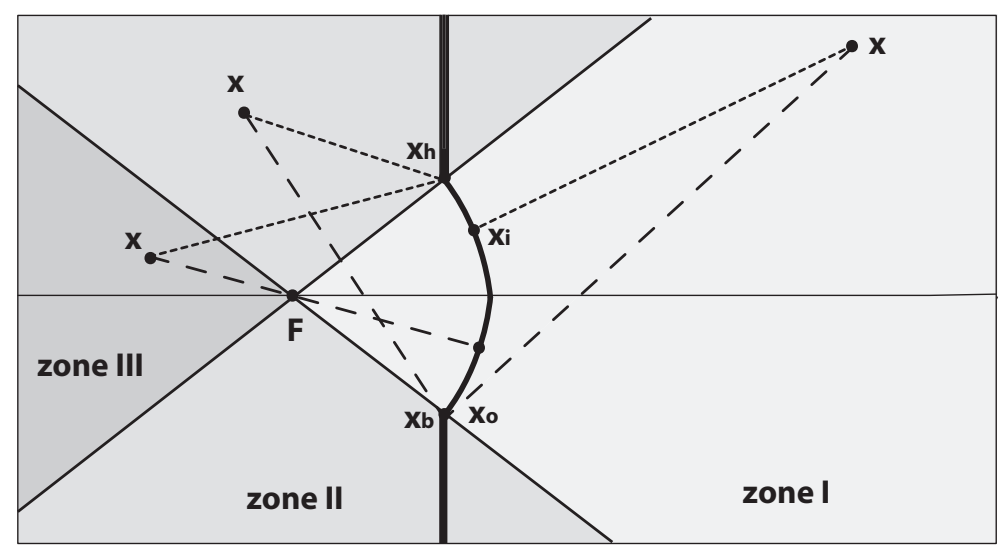

Fig. 2. Definition of the 3 different areas of calculation. For each position of the receiver, the associated points $\mathbf{x}_{\mathbf{i}}$ (dotted lines) and $\mathbf{x}_{\mathbf{o}}$ (dashed lines) are represented . The zones I and III only are defined in front and in back of a convex structure respectively. The intersection point $F$ of the 3 zones is called the sweet spot (or geometrical focal point).

bottom of the structure, as shown in Fig. 2. Depending on the structure and on the location of the receiver $\mathbf{x}$, one can define 3 different zones defined in Tab. 1, where the radiation properties differ.

Table 1

\begin{tabular}{|c||c|}
\hline zone & Conditions on $\mathbf{x}_{\mathbf{i}}$ and $\mathbf{x}_{\mathbf{o}}$ \\
\hline \hline zone I & $\mathbf{x}_{\mathbf{i}} \neq\left\{\mathbf{x}_{\mathbf{h}}, \mathbf{x}_{\mathbf{b}}\right\}$ and $\mathbf{x}_{\mathbf{o}}=\left\{\mathbf{x}_{\mathbf{h}}, \mathbf{x}_{\mathbf{b}}\right\}$ \\
\hline zone II & $\mathbf{x}_{\mathbf{i}}=\left\{\mathbf{x}_{\mathbf{h}}, \mathbf{x}_{\mathbf{b}}\right\}$ and $\mathbf{x}_{\mathbf{o}}=\left\{\mathbf{x}_{\mathbf{h}}, \mathbf{x}_{\mathbf{b}}\right\}$ \\
\hline zone III & $\mathbf{x}_{\mathbf{i}}=\left\{\mathbf{x}_{\mathbf{h}}, \mathbf{x}_{\mathbf{b}}\right\}$ and $\mathbf{x}_{\mathbf{o}} \neq\left\{\mathbf{x}_{\mathbf{h}}, \mathbf{x}_{\mathbf{b}}\right\}$ \\
\hline
\end{tabular}

Definition of the 3 calculation zones.

\subsection{Change of coordinates}

If the present section, the aim is to transform the integration variables of Eq. (5) by 2 variables $\left|\mathbf{x}-\mathbf{x}_{\mathbf{S}}\right|$ and an angle $\phi$ that correspond to the geometry of the problem.

Let $\theta_{i}$ denote the angle between axis $u_{z}$ and the line $\left(\mathbf{x x}_{\mathbf{i}}\right)$. A new local orthonormal basis $\mathcal{B}_{i}=\left(\overrightarrow{t_{i x}}, \overrightarrow{t_{i y}}, \overrightarrow{n_{i}}\right)$ centered on point $\mathbf{x}_{\mathbf{i}}$ is introduced, as presented in Fig. 3. It is defined in the Cartesian basis $\mathcal{B}_{c}=\left(\overrightarrow{u_{x}}, \overrightarrow{u_{y}}, \overrightarrow{u_{z}}\right)$ as follows:

$$
\left\{\begin{array}{l}
\overrightarrow{n_{i}}=\quad-\sin \theta_{i} \overrightarrow{u_{y}}-\cos \theta_{i} \overrightarrow{u_{z}}, \\
\overrightarrow{t_{i y}}=\quad \cos \theta_{i} \overrightarrow{u_{y}}-\sin \theta_{i} \overrightarrow{u_{z}}, \\
\overrightarrow{t_{i x}}=\overrightarrow{u_{x}} .
\end{array}\right.
$$






Fig. 3. Definition of the new local basis $\mathcal{B}_{i}=\left(\overrightarrow{t_{i x}}, \overrightarrow{t_{i y}}, \overrightarrow{n_{i}}\right)$ and the new coordinates $\left(r_{2}, \phi\right)$. The points $\mathbf{x}, \mathbf{x}_{\mathbf{i}}$ and $\mathbf{x}_{\mathbf{S}}$ denote the observation point (located in the zone I for the present example), the projection point and an integration point located on the surface $S$, respectively.

In this basis, a set of cylindrical coordinates is introduced for any point located on the surface $\mathbf{x}_{\mathbf{S}}=\left(r_{2}, \phi\right)$ and the coordinates in the new local basis are defined in appendix A. Introducing the tangent vectors to the surface $\overrightarrow{T_{\left|\mathbf{x}-\mathbf{x}_{\mathbf{S}}\right|}}$ and $\overrightarrow{T_{\phi}}$ with respect to the variables $\left|\mathbf{x}-\mathbf{x}_{\mathbf{S}}\right|$ and $\phi$ (detailed in Appendix A), the integration vector of Eq. (5) becomes in the new local basis[26]:

$$
\overrightarrow{d \mathbf{x}_{\mathbf{S}}}=\left(\overrightarrow{T_{\phi}} \times \overrightarrow{T_{\left|\mathbf{x}-\mathbf{x}_{\mathbf{S}}\right|}}\right) d\left|\mathbf{x}-\mathbf{x}_{\mathbf{S}}\right| d \phi .
$$

where $() \times.($.$) denotes the cross product between 2$ vectors. The expression of tangent vectors and integration vector can be found in Appendix A. The principle is to replace the integration vector in Eq.(5) by the new one defined in Eq. (8) and to integrate over $\left|\mathbf{x}-\mathbf{x}_{\mathbf{S}}\right|$ in order to eliminate the Dirac delta function contained in the Green's function (using the convolution theorem [27]). Introducing the time delay $\tau=t-t^{\prime}$, one obtains the final formulation of the Rayleigh integral:

$$
P(\mathbf{x}, t)=-\frac{\rho c}{2 \pi} \int_{0}^{t} \sum_{p} \mathcal{H}_{p}(\mathbf{x}, \tau) \ddot{q}_{p}(t-\tau) d \tau
$$

where:

$$
\mathcal{H}_{p}(\mathbf{x}, \tau)=\left.\int_{\phi} \frac{\Phi_{p}(r)}{F A+B \cos \phi-C \sin ^{2} \phi}\right|_{\left|\mathbf{x}-\mathbf{x}_{\mathbf{S}}\right|=c \tau} d \phi
$$


and:

$$
\left\{\begin{array}{l}
F=1+\frac{d h_{0}}{d r}\left(\frac{h_{0}(r)-z}{r}\right) \\
A=\left(\frac{1-\left(\sin \theta_{i} \sin \phi\right)^{2}}{\cos \theta_{i}}\right) \\
B=\left(\frac{F r_{i}-y}{r_{2}}\right) \\
C=\left(\frac{d h_{0}}{d r}\left(\frac{y}{r}\right) \sin \theta_{i}\right)
\end{array}\right.
$$

In this expression, the Spatial Impulse Response explicitly depends on the profile function $h_{0}(r)$, the modal shape $\Phi_{p}(r)$ (also dependent on the shape of the source), the position of the receiver $\mathbf{x}$ and the integration time $\tau$. The notation $\left|\mathbf{x}-\mathbf{x}_{\mathbf{S}}\right|=c \tau$ means that for each time $\tau$, a distance $\left|\mathbf{x}-\mathbf{x}_{\mathbf{S}}\right| / c$ is associated for computing the integrand of Eq. (10). In that case, for each time step $\tau$, the intersection between the structure and the sphere centered on observation point $\mathbf{x}$ and of radius $c \tau$ is sought.

This path (determined by the boundary values of integration path over $\phi$ ) is unique in the case of a monotonic profile function (when the sign of $\dot{h_{0}}(r)$ is constant) and can be closed if $\phi=[0: 2 \pi]$ or open if $\phi=\left[\phi_{\min }: \phi_{\max }\right]$ depending on the location of the receiver, the shape of the structure and the integration time $\tau$. The calculation of those boundaries and the application in the case of a spherical cap are expressed in Appendix B for each zone of calculation.

The formulation of Eq. (10) allows to reduce the order of integration compared to Eq. (5): only one spatial coordinate on variable $\phi$ is required and since spatial and time-domain integration are separated, the spatial discretization is independent on the time step (and thus the maximal simulated frequency). In Fig. 4, the Spatial Impulse Response is computed for a hemispherical shell and a receiver located at $(y, z)=(a, a)$ i.e. in the near-field region and for different discretization steps on variable $\phi$ in Eq. (10). The solutions in each case is compared to the solution of the Rayleigh integral [13] represented by points. It appears that less than 20 points for $\phi$ are required to attain a convergence error below $1 \%$ of the integral defined by Eq. (10). 


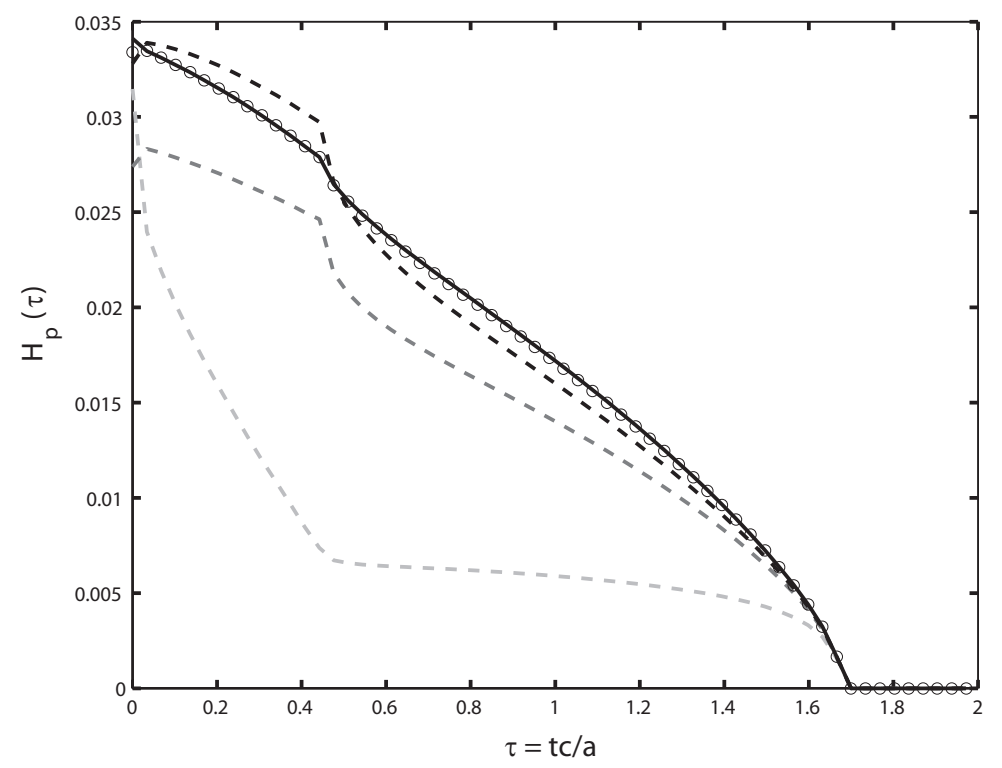

Fig. 4. Spatial Impulse Responses in the case of a hemispherical transducer and for a receiver located at $(y, z)=(a, a)$. The exact solution of the Rayleigh's integral in the time-domaine derived from [13] is represented by points ( $\circ \circ \circ)$ and the results obtained using Eq. (10) in the case of different discretizations of the integral over $\phi$ are represented by lines : 3 points (dashed light grey line), 6 points (dashed dark grey line), 9 points (dashed black line) and 12 points (solid black line). The convergence is ensured in any case with at less 20 points.

\section{VALIDATION}

\subsection{Planar sources}

In the case of a planar structure, the profile function is equal to zero: $h_{0}(r)=0$. In that case, Eq. (10) becomes [7]:

$$
\mathcal{H}_{p}(\mathbf{x}, \tau)=\left.\int_{\phi} \Phi_{p}(r)\right|_{\left|\mathbf{x}-\mathbf{x}_{\mathbf{S}}\right|=c \tau} d \phi
$$

with the boundaries derived from Eq. (C.1) in Appendix B in accordance with the results of Harris [6] and Jensen[8]. The classical example [6] of Spatial Impulse Responses obtained for a planar piston with a uniform velocity distribution is shown in Fig. 5. In this figure, S.I.R. functions are calculated using an integral discretization of 20 points. The in-axis impulse response is equal to 1 in a compact support which means that the effect of radiation filter is equivalent to a low pass filter whose cut-off frequency is determined by the size of the support of the Spatial Impulse Response. The off-axis response decreases with respect to the off-axis position $y$ while its compact support increases. In that case, the radiation filter is also equivalent to a low pass filter whose cut-off frequency is below the the in-axis cut-off frequency. This result 


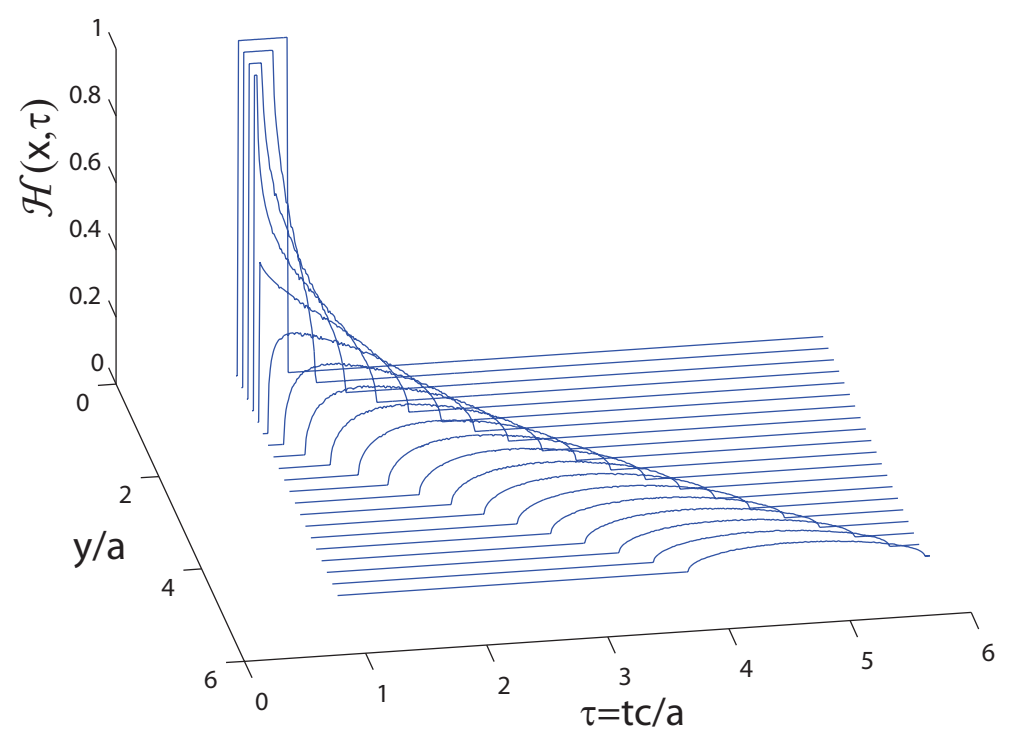

Fig. 5. Spatial Impulse Responses obtained in the case of a plane piston with an uniform velocity distribution. The solutions are computed for a receiver located in $z=a$ and an off-axis position $y$ from 0 to $5 a$ and for 30 points of discretization in Eq. (12).

is general for axisymmetric structures : the radiation filter is equivalent to a low-pass filters whose cut-off frequency decreases with respect to the off-axis position $y$.

\subsection{Spherical sources}

In the present section, the approximated formulation of Eq. (9) is compared to the numerical results obtained by Suzuki [22] using 40 spherical harmonics in the wavenumbers domain, taking into account the diffraction effects. In the case of spherical sources, the profile function $h_{0}(r)$ equals:

$$
\begin{cases}h_{0}(r)=\sqrt{R^{2}-r^{2}}-\sqrt{R^{2}-a^{2}} & \text { for } r<a, \\ h_{0}(r)=0 & \text { for } r>a,\end{cases}
$$

where $R$ denotes the radius of curvature of the spherical shell and $a$ the external radius of the structure. In order to compare equivalent results in the frequency domain, one needs to take the Fourier transform of the Spatial Impulse Response computed using Eq. (10) in the case of a rigid body motion (one mode of vibration: $p=1$ and uniform displacement of the structure: $\Phi_{p}(r)=1$ for $\left.r<1\right)$. In the work of Suzuki, the calculations are limited by the order of the spherical harmonics (40 for his study), so that his model is restricted to wavenumbers up to $k a=10$. In the present formulation, only the time discretization of Spatial Impulse Responses influences the upper $k a$ 

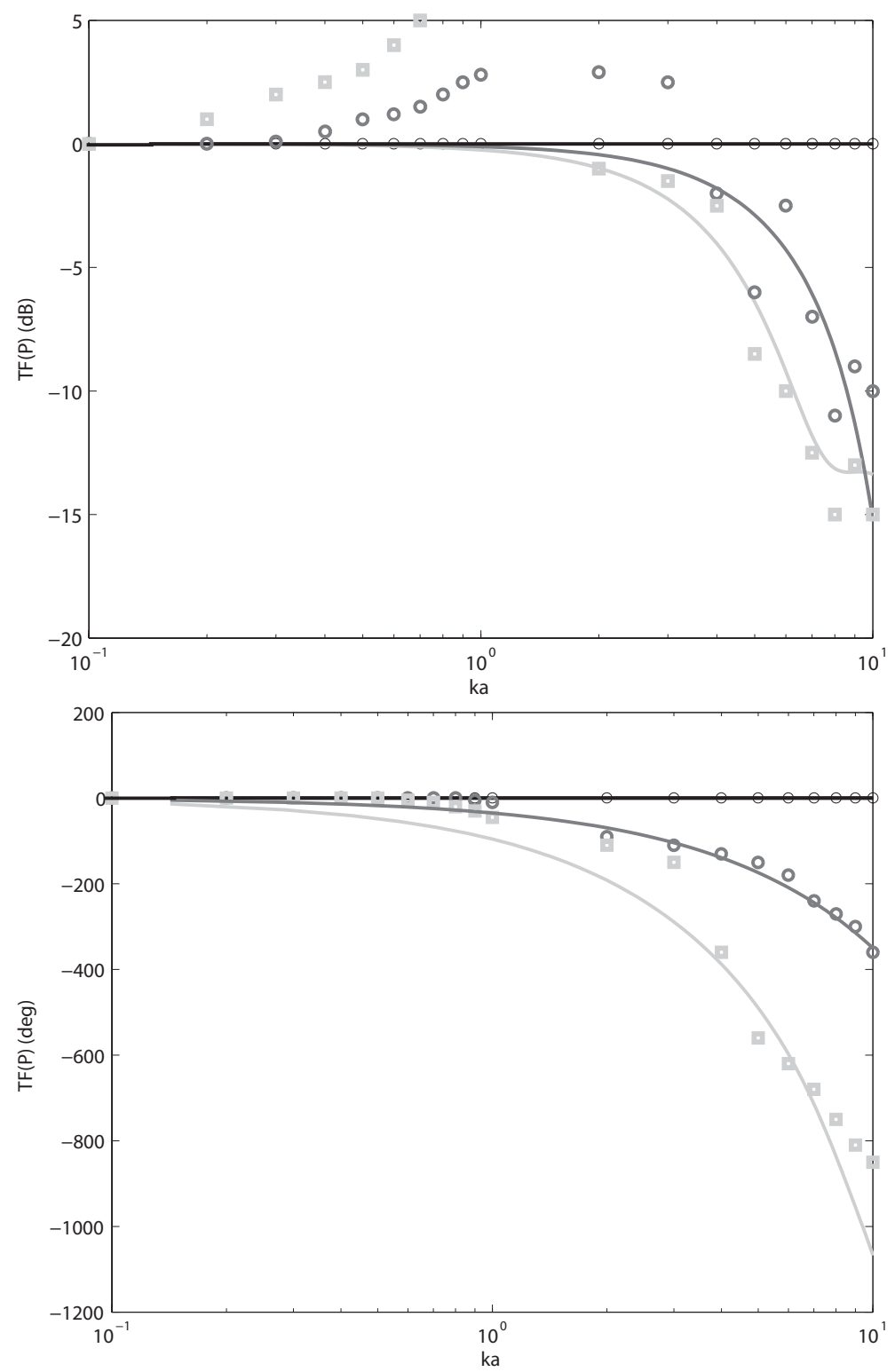

Fig. 6. On-axis acoustic pressure (up: magnitude in $\mathrm{dB}$, down: phase in degrees) in far-field versus nondimensionalized wavenumber $k a$ computed by Suzuki[22] (points) and using Eq. (9) (lines). The results are presented for 3 different concave structures: planar piston (black), medium spherical cap (dark grey) and hemispherical cap (light gray). The wavenumbers domain results are obtained by taking the Fourier transform of the Spatial Impulse Response computed in the time domain.

limit, so that no limitation of frequency range is observed. In practical cases, the Spatial Impulse Responses are computed using 20 nondimensionnalized time steps $\tau=t c / a$ and then completing with zeros, the precision in the wavenumbers domain is adaptable.

The results are presented for 3 different structures: a planar piston, a spherical cap defined by a radius of curvature $R=1.5 a$ and an hemispherical cap 

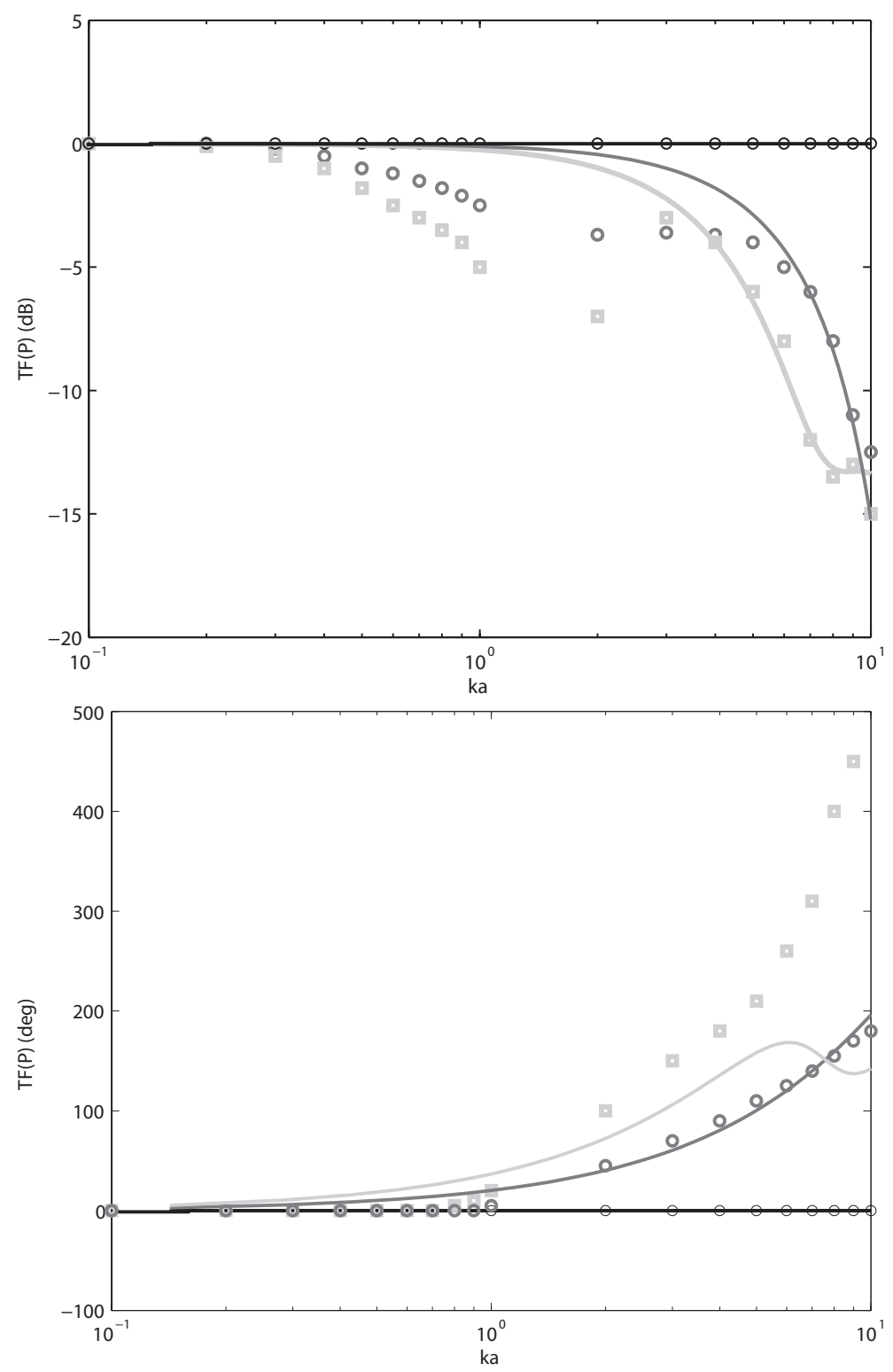

Fig. 7. On-axis acoustic pressure (up: magnitude in $\mathrm{dB}$, down: phase in degrees) in far-field versus wavenumber $k a$ computed by Suzuki[22] (points) and using Eq. (9) (lines). The results are presented for 3 different convex structures: planar piston (black), medium spherical cap (dark grey) and hemispherical cap (light gray).

defined by a radius of curvature $R=a$. The Fourier Transform of the Spatial Impulse Responses computed for an on-axis receiver located in the far-field $(y, z)=(0,100 a)$ are presented in Figs. 6 and 7 for concave and convex caps and a good agreement between the present approach and the results of Suzuki is obtained for low wavenumbers $(k a<1)$ and high wavenumbers $(k a>3)$.

For low wavenumbers $(k a<1)$, the monopole approximation is valid and the equivalent radiation filter has a flat response. For high wavenumbers $(k a>3)$, 


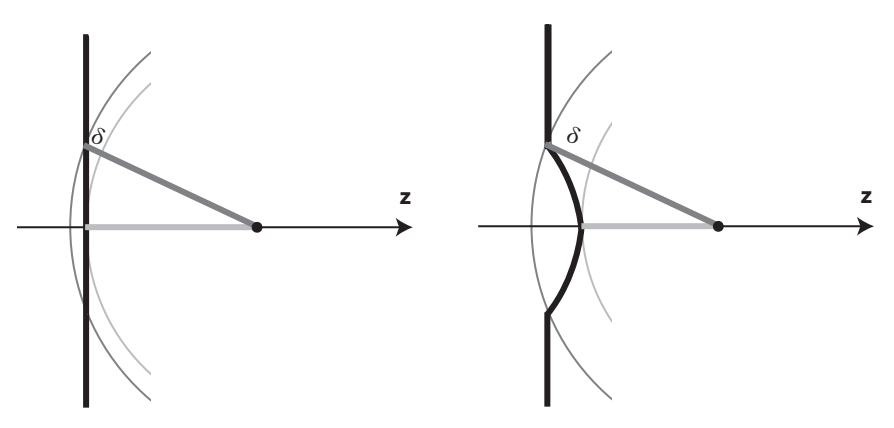

Fig. 8. Explanation of the interference phenomenon that appears when the wavelength $k$ is above the path difference between the nearest and the farther point seen from the observer point. In the frequency domain, this effect is responsible for a slope of the on-axis sound pressure level of $-20 \mathrm{~dB} /$ decade and an extension of the directivity compared to planar radiators.

the local curvature induces a decrease of the on-axis sound pressure level and the radiation filter is thus equivalent to a low-pass filter with a slope of $20 \mathrm{~dB} /$ decade above a cut-off wavelength depending on the curvature $R$ of the profile and its radius $a$. In Figs. 6 and 7 the cut-off frequency of the equivalent low-pass filter decreases when the curvature radius $R$ increases. This is explained by interferences phenomena that appears when the wavelength $\lambda=1 / k$ is below the path difference between the nearest and the farther point seen from the observer point as presented in Fig. 8. Thus the more the structure radius $R$ is important, the more the difference path increases and the more the cut-off frequency of the equivalent low-pass filter decreases.

Around $k a=1$, the diffraction taken into account in the calculations of Suzuki [22] induces an increase of on-axis sound pressure level up to $+6 \mathrm{~dB}$ in the case of concave caps (a decrease in the case of convex structures) that is not taken into account in the present model. The effect of diffraction appears in the non-dimensionnalized wavenumbers domain $1<k a<3$ i.e. when the wavelength approaches the maximal depth of the structure. The influence of the diffraction is hardly dependent on the shape and the validity of the present approach is thus no longer viable in the wavelength range $1<k a<3$.

Figs. 9 and 10 represent the directivity patterns in linear scale computed for the considered spherical caps in the far-field $(z=100 a)$ for wavenumbers such as $k a=1, k a=3$ and $k a=10$. Again, the results obtained by taking the Fourier Transform of Eq. (10) are compared to results obtained by Suzuki [22]. For low wavenumbers $(k a=1)$ the radiation patterns are almost omnidirectional and become more directive for high wavenumbers $(k a>3)$. The curvature extends the directivity in both convex and concave cases and in the concave case, the maximum of sound pressure is obtained for a precise obser- 
vation angle depending on the curvature of the considered shell.

In a general manner, the curvature (not necessarily in the case of a spherical shell) induces a decrease of on-axis sound pressure field due to interference for high wavenumbers. The effects of diffraction, not taken into account in the present model, are responsible for an increase of on-axis sound pressure level around $1<k a<3$ in the case of concave sources (a decrease in the case of convex structures). The on-axis high wavenumbers losses due to interferences are compensated by an extent of directivity related to the local curvature of the source. This result is general for monotonic profile functions and is extended in the following section in the case of non-monotonic profile function and in the case of a typical loudspeaker shape.

\subsection{Complex sources}

The previous formulation is only valid for a monotonic profile function $h_{0}(r)$. In the case of a nonmonotonic profile, i.e. when the sign of $\dot{h_{0}}(r)$ is not constant, the integration path (intersection between the sphere centered on observation point $\mathbf{x}$ and of radius $c \tau$ used in the calculation of Eq. (10)) is made of multiple paths corresponding to each monotonic part of the global profile.

Neglecting the multiple reflections over the emissive surface, the global radiation can be expanded as the sum of contributions of each monotonic portion of the profile. For example, for a global structure composed by 2 monotonic profiles $S=S_{1} \cup S_{2}$, we have:

$$
\begin{array}{r}
P(\mathbf{x}, t)=-\frac{\rho c}{2 \pi} \sum_{p}\left(\int_{0}^{t} \mathcal{H}_{p}^{1}\left(\mathbf{x}, \tau_{1}\right) \ddot{q}_{p}\left(t-\tau_{1}\right) d \tau_{1}\right. \\
\left.+\int_{0}^{t} \mathcal{H}_{p}^{2}\left(\mathbf{x}, \tau_{2}\right) \ddot{q}_{p}\left(t-\tau_{2}\right) d \tau_{2}\right)
\end{array}
$$

where $\mathcal{H}_{p}^{i}\left(\mathbf{x}, \tau_{1}\right)$ corresponds to the Spatial Impulse Response of mode $p$ associated to the $i^{\text {th }}$ monotone part of the profile. This procedure is applied in the case of a typical loudspeaker shape composed as the junction of a truncated cone with a spherical cap. The profile function is then described by:

$$
\begin{cases}h_{0}(r)=\alpha(b-a)+\sqrt{R^{2}-r^{2}}-\sqrt{R^{2}-b^{2}} & \text { for } r<b, \\ h_{0}(r)=\alpha(r-a) & \text { for } r>b, \\ h_{0}(r)=0 & \text { for } r>a,\end{cases}
$$

where $R$ denotes the curvature of the inner spherical shell, $b$ the radius of the 



$\mathrm{ka}=3:$

0



Fig. 9. Directivity functions (using a linear scale) for the 3 different concave caps: plane piston (black), medium spherical cap (dark grey) and hemispherical cap (light gray). The results obtained by the present model (right) are compared to the results obtained by Suzuki [22] (left) for 3 wavenumbers: $k a=1$ (up), $k a=3$ (middle) and $k a=10$ (bottom).

junction between both sub-structures and $\alpha$ the depth of the truncated conical shell. The following results are obtained for a typical loudspeaker $\alpha=1$, $b=0.5 a$ and $R=1.5 b$. The profile function and the principle of calculation of the on-axis Spatial Impulse Response are presented in Fig. 11 and the onaxis result in the nondimensionalized wavenumbers domain $k a$ is displayed in 



$\mathrm{ka}=3:$

0

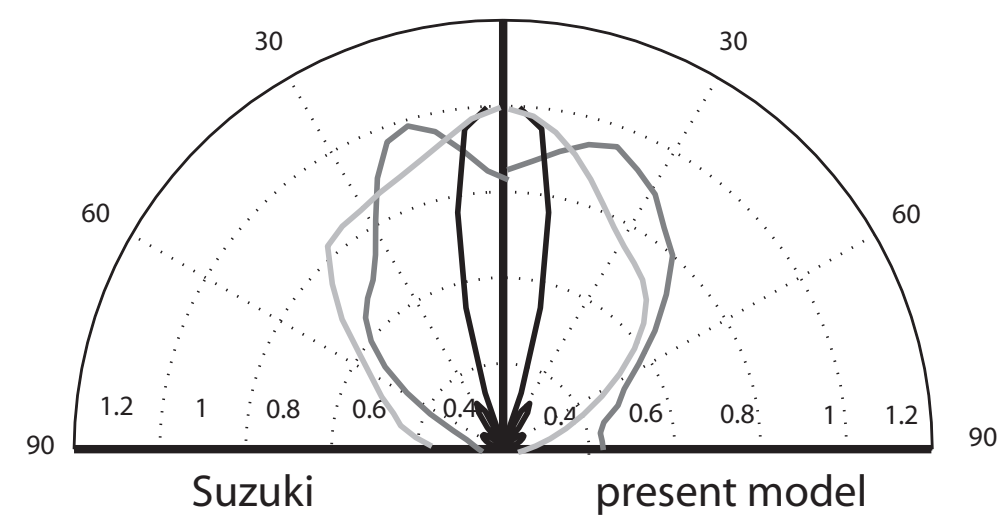

Fig. 10. Directivity functions (using a linear scale) for the 3 different convex caps: plane piston (black), medium spherical cap (dark grey) and hemispherical cap (light gray). The results obtained by the present model (right) are compared to the results obtained by Suzuki [22] (left) for 3 wavenumbers: $k a=1$ (up), $k a=3$ (middle) and $k a=10$ (bottom).

Fig. 12 taking the Fourier Transform of computed time function.

In Fig. 11, the respective contribution of each sub-structure in the time-domain for an on-axis receiver is represented. In the frequency domain, the global ra- 


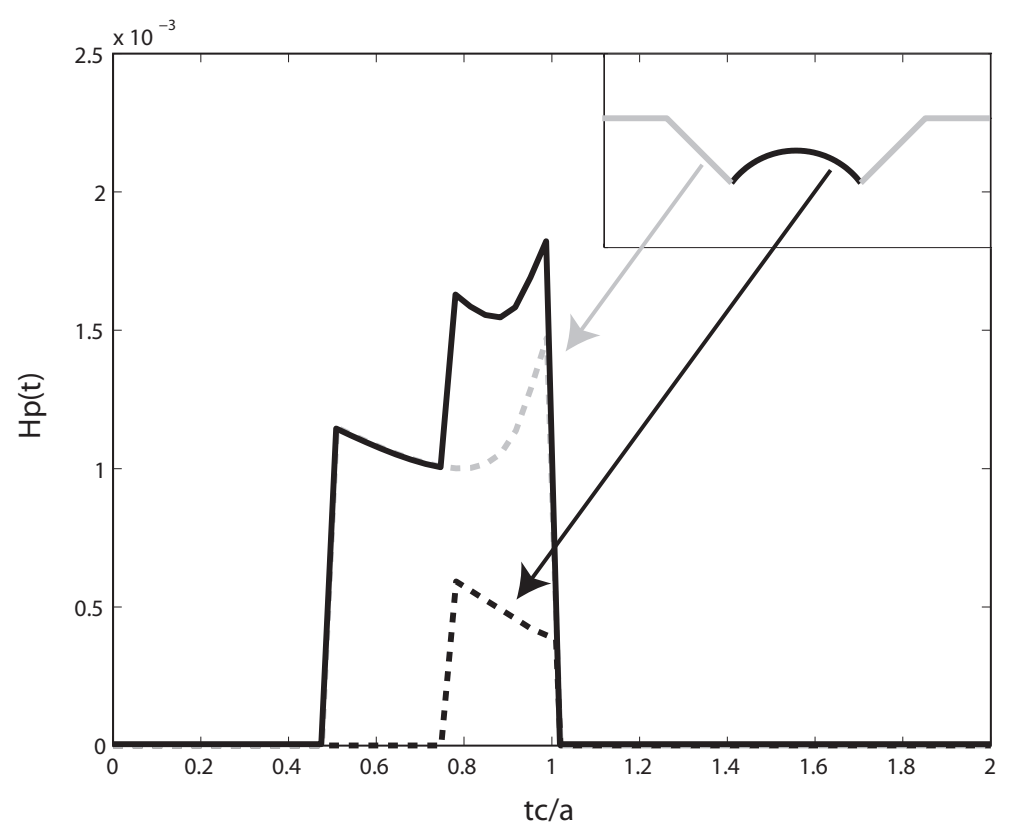

Fig. 11. Calculation procedure of the Spatial Impulse Response of a typical loudspeaker (junction of a cone and a spherical cap) for an uniform velocity distribution and for a on-axis receiver located in $z=a$. The S.I.R. corresponding to the spherical cap $\mathcal{H}^{1}(\mathbf{x}, \tau)$ is represented by a dashed black line, the S.I.R. corresponding to the truncated cone $\mathcal{H}^{2}(\mathbf{x}, \tau)$ is represented by a dashed grey line and the global S.I.R. corresponds to the solid black line.

diation filter is as previously equivalent to a low-pass filter whose cut-off frequency is not only related to the depth of the truncated conical shell. Indeed, between $k a=1$ and $k a=10$ in Fig. 12, the comparison between the response of the truncated conical shell only and the global structure shows that the cutoff frequency is increased when the inner spherical cap is present. The effect of the convex spherical cap compensate the interference effect around $k a=1$ and an extension of the flat acoustic response is achieved and a global increase of $+3 \mathrm{~dB}$ with respect to the conical source is observed in the interference regime.

The contribution of the inner cap appears in the tail of the Impulse Response and is also responsible for high wavenumber comb-filtering (for $k a>10$ ) that is not present in absence of the inner spherical cap (see Fig. 12). Around $k a=25$, a decrease of $10 d B$ with respect to the conical source is observed and can be interpreted as interferences between the conical and the spherical cap because this phenomenum is not present in the case of a spherical shell only neither a conical shell only as presented on Fig. 12. This effect is then amplified above $k a=25$ and is responsible for comb filtering at multiples of that nondimensionalized wavenumber $k a=25, k a=50$ and $k a=75$. 


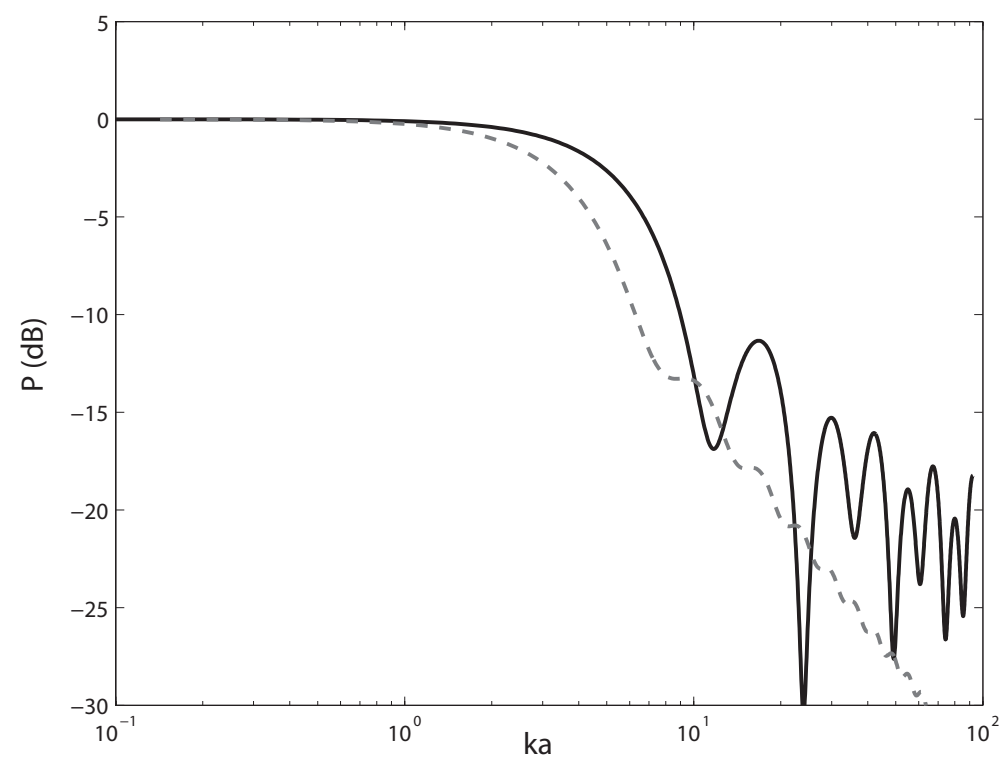

Fig. 12. On-axis acoustic pressure level (magnitude in $\mathrm{dB}$ versus wavenumber $k a$ ) obtained in the case of a loudspeaker (junction of a truncated cone and an hemispherical cap). The solution (solid black line) is compared to the truncated cone radiation only (dashed grey line). The use of a typical loudspeaker profile increases the low-pass filter cut-off frequency and induces oscillations in the on-axis acoustic pressure level, due to complex interference phenomena.

\section{APPLICATION TO A PROTOTYPE OF LOUDSPEAKER}

\subsection{Presentation}

In order to illustrate the influence of the diaphragm shape of a loudspeaker on its acoustical response for high amplitudes of vibration, a prototype of loudspeaker has been developed by G. Lemarquand in the LAUM [25] as presented on Fig. 13. Its moving part is an aluminium convex spherical shell of $50 \mathrm{~mm}$ of diameter. Experiments have been done with a curvature equals to $a / R=0.3$ which is defined as the ratio between the external radius and the radius of curvature of the shell. For the prototype, the moving part is not made in one piece, since the emissive surface is glued on the supporting cylinder.

In the studied prototype, attention is paid to the motor and suspensions, in order to minimize the "electrical" nonlinear phenomena. The sources of nonlinearities in the motor are the variations of the fore factor $(B l)$, and the Eddy currents. As a remedy to these defects, an ironless motor was used, which is made only out of neodymium iron boron permanent magnets. The classical suspensions of loudspeakers are mostly made of rubber, impregnated fabric or molded plastic. They act as a spring, but have a nonlinear behavior. 




Fig. 13. Photography of the studied prototype in anechoic room.

This means that their compliance depends on the movements amplitude and, above all, that the induced damping depends greatly on both amplitude and frequency. These phenomena are the sources of the classical nonlinear behavior of the loudspeakers suspensions at low frequencies. In the studied prototype, the almost perfect compressibility properties of the air were used to create a pneumatic stiffness. Indeed, the air is compressed in the closed box, whose volume tunes the stiffness value. The cabinet is thus a cylindrical pipe which is closed and filled up with an absorbing material. Classical electrical nonlinearities are thus not observed in the present prototype and only geometrical nonlinearities remain and are due to large amplitudes of vibration of the moving part. This kind of nonlinearities has been pointed out mechanically but the influence on the radiated sound pressure field had not been cleared explained.

\subsection{Electromechanical characterization}

The first step of the experimental analysis consist in understanding and modeling the electromechanical behavior of the prototype. Fig. 14 represents the impedance curves in both magnitude. Below $1 \mathrm{kHz}$, the behavior is mostly resistive and become inductive above $2 \mathrm{kHz}$. As mentioned above, the inductive part is not modified by eddy currents, due to the ironless conception of the motor, and then a linear dependency of impedance magnitude with respect to frequency is observed (not observable on Fig.14 because of the logarithmic scale for the frequencies).

However, the impedance curves are affected by resonances (amplification of certain frequencies and perturbation in the phase curve) of different parts of 

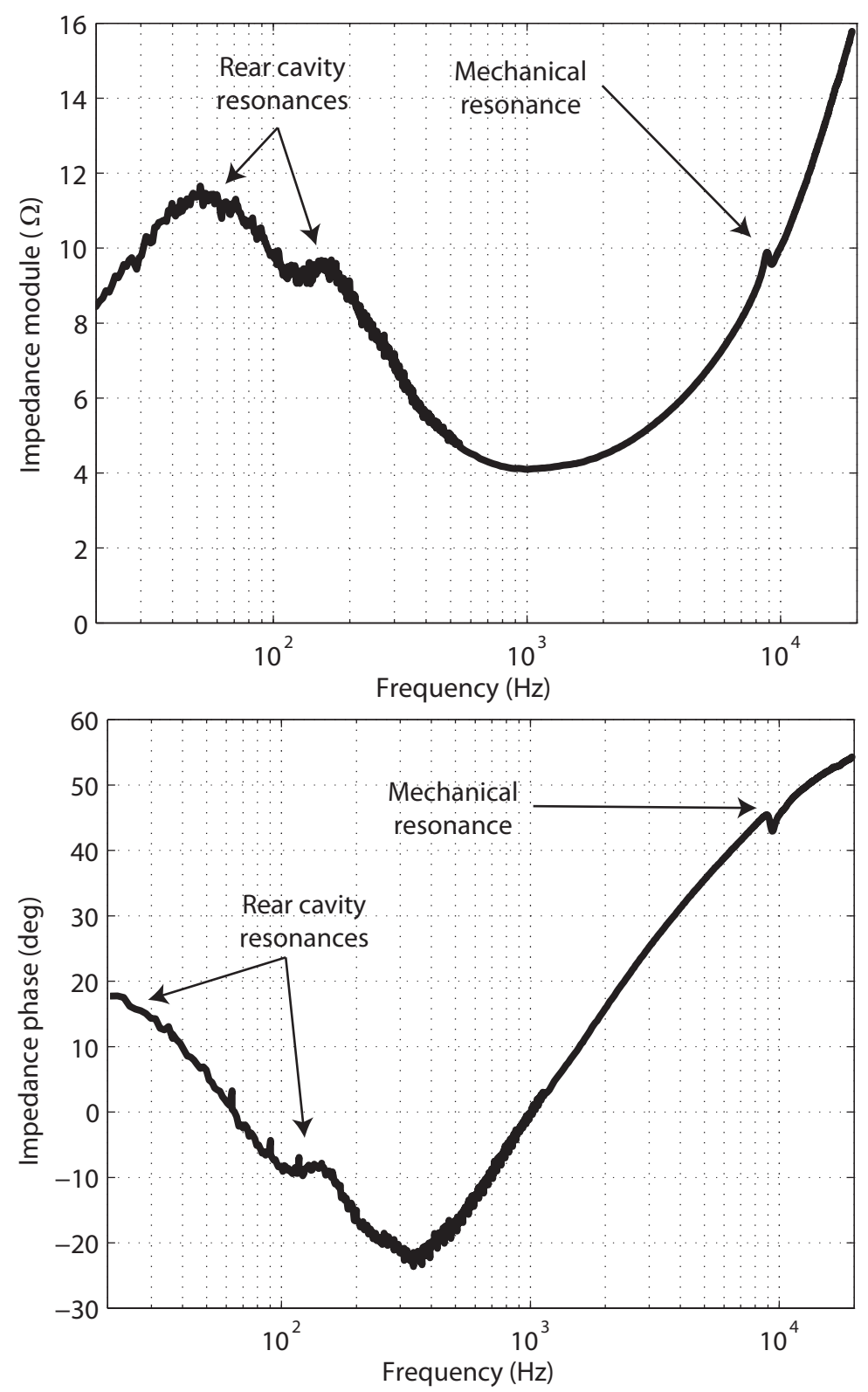

Fig. 14. Electrical impedance of the loudspeaker in magnitude (up) and phase (down).

the prototype. Two tendencies are easily identified on Fig. 14 :

- for frequencies below $5 \mathrm{kHz}$, the resonances are due to stationary waves in the rear cavity : the first resonance is around $70 \mathrm{~Hz}$. Under this assumption, the vibration pattern is then assimilated to a plane piston (rigid body mode).

- for frequencies above $9 \mathrm{kHz}$, the resonances correspond to mechanical reso- 
nances of the spherical cap, as described in the following part. The properties of those resonances (eigenfrequencies, modal damping, excitation and shapes) have been analyzed using Laser Doppler Velocity and a linear modal analysis software $\left(\right.$ IDEAS $^{\circledR}$ ) and are presented on Tab. 2.

\begin{tabular}{|c|c|c|c|c|}
\hline $\begin{array}{c}\text { Mode } \\
p\end{array}$ & $\begin{array}{c}\text { Resonance } \\
\text { Frequency }(\mathrm{Hz})\end{array}$ & $\begin{array}{c}\text { Modal } \\
\text { Damping(\%) }\end{array}$ & $\begin{array}{c}\text { Excitation } \\
\text { Vector }\end{array}$ & $\begin{array}{c}\text { Modal } \\
\text { Shape } \Phi_{p}\end{array}$ \\
\hline 1 & 73 & 100 & 1 & \\
\hline 2 & 9650 & 3.2 & 20 & \\
\hline 3 & 17290 & 0.6 & 2 & \\
\hline 4 & 18550 & 0.6 & 0.8 & \\
\hline
\end{tabular}

Table 2

Axisymmetric modal parameters (eigenfrequency $f_{p}$, modal damping $\mu_{p}$ and excitation $T_{p}$ associated to mode $p$ ) measured for the spherical dome. The first mode corresponds to a piston mode (rear cavity resonance) and is very damped compared to the structural modes (above $9 \mathrm{kHz}$ ).

\subsection{Nonlinear radiation : on-axis response}

For high amplitudes of vibration, nonlinearities appear and give rise to harmonic distortion in the velocity and sound pressure signals. Due to the ironless conception of the motor, the electrical nonlinearities classically observed in electrodynamic loudspeakers vanish and only geometrical nonlinearities (due to large deformations of the moving part) remain.

This type of nonlinearities can be modeled using the modal formulation proposed in [5]. This approach is absolutely suitable in the present case, since 
few modes radiate in the audible range. The principle is to model the prototype as a 4 degrees of freedom system (since 4 modes radiate in the audible range, as presented in Tab. 2) and to include geometrical nonlinearities in the modal equations through cubic and quadratic nonlinearities [2]. In order to illustrate the typical effects of geometrical nonlinearities on radiation of the prototype, only quadratic and cubic nonlinearity terms have been added for the second mode of vibration (at $9650 \mathrm{~Hz}$ ). The displacement field is calculated for high amplitudes of vibrations using the State-Space formulation described by Eq. (2), and the radiation problem is solved using the Spatial Impulse Response (S.I.R.) approach developed in the present study. In this practical case, the measured modal shapes $\Phi_{p}\left(r^{\prime}\right)$ and profile function $h_{0}\left(r^{\prime}\right)$ are introduced in Eq. (10) and convolutions are performed at the sampling frequency of $48 \mathrm{kHz}$. It is important to notice that the calculations of the displacement field and radiated sound pressure are computed directly in the time-domain and the results are presented in the frequency domain for clarity (taking the Fourier transform of the transient responses).

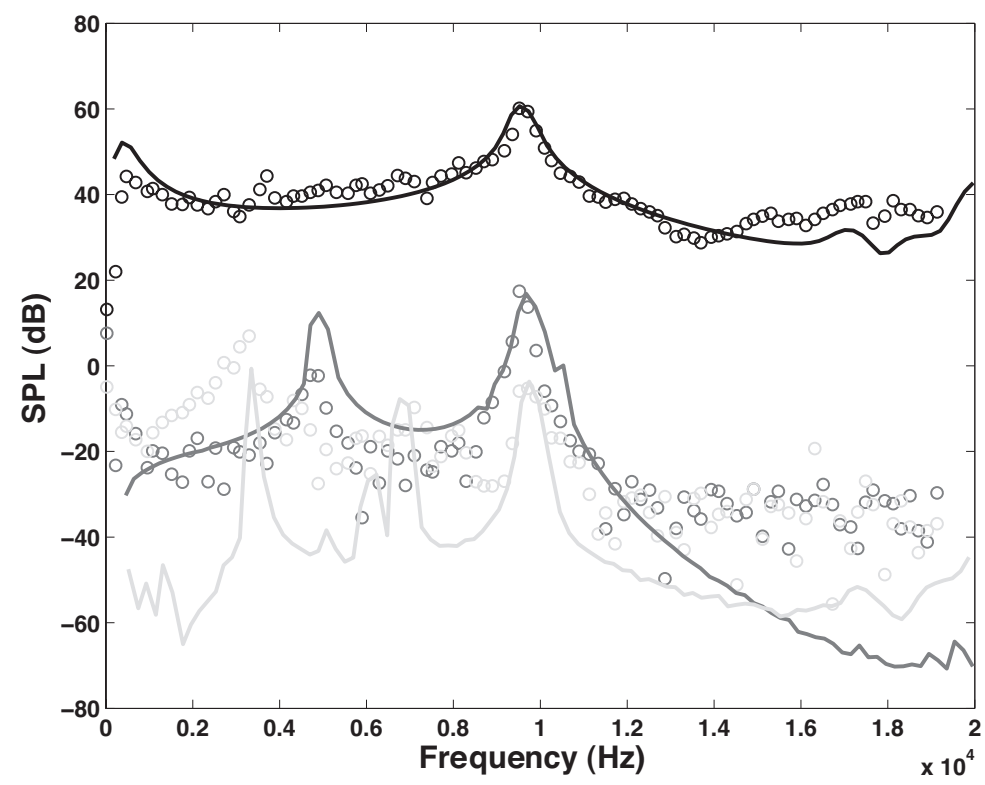

Fig. 15. Measurement (points) and prediction (solid lines) of on-axis sound pressure level for a $10 \mathrm{~W}$ input. For each frequency, the energy of fundamental (black) and harmonics 2 (dark grey) and 3 (light grey) are measured and predicted.

Fig. 15 represent the on-axis sound pressure measurement and prediction at $1 \mathrm{~m}$ of the fundamental and of the harmonics 2 and 3 in the case of the convex spherical dome. Measurements have been performed in anechoic room (approximate size $1000 \mathrm{~m}^{3}$ ) using sinusoidal input signals. A good agreement is observed in the audible bandwidth and the geometrical nonlinearities are correctly predicted around discrete frequencies such as : 
- $f=f_{a}$ : around a mechanical resonance frequency, the amplitude of vibration increases and harmonics are generated. For example, on figure 15, harmonics 2 and 3 are generated for an excitation frequency around $9.6 \mathrm{kHz}$ (first mechanical resonance).

- $f=f_{a} / p$ with $p$ integer : for the submultiple $p$ of a resonance frequency, the frequency of harmonic $p$ corresponds to an eigenfrequency and this harmonic is then amplified. For example, on Fig. 15, an increase of harmonic 3 at 3.2 $\mathrm{kHz}$ which corresponds to a third of the first mechanical resonance is observed (the same effect appears with harmonic 2 at $4.8 \mathrm{kHz}$ ).

The Spatial Impulse Response appear to model efficiently the complex radiation pattern for high amplitudes of vibration. The influence of the geometrical nonlinearities on the acoustic response of the prototype has been observed experimentally and modeled properly using the present approach. Other sources of nonlinearities could also be taken into account in order to predict the nonlinear acoustic response of classical loudspeakers.

\section{CONCLUSIONS}

In order to compute acoustic radiation from axisymmetric structures subjected to large amplitude motion, an explicit integral formulation is derived for predicting the modal time-domain radiation. The radiated acoustic pressure is assumed to be expressed as an integration of a simple source distribution (Rayleigh integral), which is valid except in the nondimensionalized wavenumber range $1<k a<3$ because the diffraction effects are neglected in the present study and will be detailed in a future work. The displacement is expanded onto the linear modes of the structure and the acoustic pressure is expressed as a sum of modal contributions, by introducing the Spatial Impulse Responses depending on the shape of the source, the position of the receiver and the modal shapes (also dependent of the shape of the source). The solution is compared to the literature in the cases of a piston and of spherical caps and gives quite good results and low calculation costs compared to frequency-domain formulations. In a general way, the radiation induces a lowpass filtering whose cut-off frequency is related to the radius of the source and the local curvature. The method is extended for predicting the radiation of an axisymmetric source whose profile function is complex and corresponds to a typical loudspeaker shape. Finally, predictions and experimental measurements of on-axis radiation of a prototype loudspeaker subjected to large amplitudes motion are compared and a good agreement is achieved on the audible bandwidth. 


\section{A Definition of the new local basis}

In the new local basis $\mathcal{B}_{i}=\left(\overrightarrow{t_{i x}}, \overrightarrow{t_{i y}}, \overrightarrow{n_{i}}\right)$, the coordinates of point $\mathbf{x}_{\mathbf{S}}$ are defined by:

$$
\begin{aligned}
\overrightarrow{\mathbf{x}_{\mathbf{i}} \mathbf{X}_{\mathbf{S}}}= & \left(\frac{h_{0}\left(r_{i}\right)-h_{0}(r)}{\cos \theta_{i}}-r_{2} \cos \phi \tan \theta_{i}\right) \overrightarrow{n_{i}} \\
& +r_{2} \cos \phi \overrightarrow{t_{i y}}+r_{2} \sin \phi \overrightarrow{t_{i x}} .
\end{aligned}
$$

where $r_{2}$ is defined by the Cartesian coordinates of integration point $\mathbf{x}_{\mathbf{S}}=$ $(x, y)$ :

$$
r_{2}^{2}=x^{2}+\cos ^{2} \theta_{i}\left[y-r_{i}-\left(h_{0}(r)-h_{0}\left(r_{i}\right)\right) \tan \theta_{i}\right]^{2} .
$$

Under those considerations, the tangent vectors to the surface $\overrightarrow{T_{\left|\mathbf{x}-\mathbf{x}_{\mathbf{S}}\right|}}$ and $\overrightarrow{T_{\phi}}$ with respect to the variables $\left|\mathbf{x}-\mathbf{x}_{\mathbf{S}}\right|$ and $\phi$ are described by:

$$
\left\{\begin{array}{l}
\overrightarrow{T_{\left|\mathbf{x}-\mathbf{x}_{\mathbf{S}}\right|}}=\frac{\partial \overrightarrow{\mathbf{x}_{\mathbf{S}}} \cdot \overrightarrow{u_{x}}}{\partial\left|\mathbf{x}-\mathbf{x}_{\mathbf{S}}\right|} \overrightarrow{u_{x}}+\frac{\partial \overrightarrow{\mathbf{x}_{\mathbf{S}}} \cdot \overrightarrow{u_{y}}}{\partial\left|\mathbf{x}-\mathbf{x}_{\mathbf{S}}\right|} \overrightarrow{u_{y}}+\frac{\partial \overrightarrow{\mathbf{x}_{\mathbf{S}}} \cdot \overrightarrow{u_{z}}}{\partial\left|\mathbf{x}-\mathbf{x}_{\mathbf{S}}\right|} \overrightarrow{u_{z}}, \\
\overrightarrow{T_{\phi}}=\frac{\partial \overrightarrow{\mathbf{x}_{\mathbf{S}}} \cdot \overrightarrow{u_{x}}}{\partial \phi} \overrightarrow{u_{x}}+\frac{\partial \overrightarrow{\mathbf{x}_{\mathbf{S}}} \cdot \overrightarrow{u_{y}}}{\partial \phi} \overrightarrow{u_{y}}+\frac{\partial \overrightarrow{\mathbf{x}_{\mathbf{S}}} \cdot \overrightarrow{u_{z}}}{\partial \phi} \overrightarrow{u_{z}}
\end{array}\right.
$$

\section{B Change of variable}

One point located in the integration surface $S$ is parameterized in the Cartesian basis by its 3 coordinates $\mathbf{x}_{\mathbf{S}}=\left(x, y, h_{0}\left(\sqrt{x^{2}+y^{2}}\right)\right)$. In order to compute the tangent vectors expressed in Eq. (A.3), one needs to express the dependency of $\left|\mathbf{x}-\mathbf{x}_{\mathbf{S}}\right|$ and $\phi$ with respect to those 3 coordinates:

$$
\left\{\begin{array}{l}
\frac{\partial x}{\partial\left|\mathbf{x}-\mathbf{x}_{\mathbf{S}}\right|}=\frac{\left|\mathbf{x}-\mathbf{x}_{\mathbf{S}}\right|}{F x} \\
\frac{\partial y}{\partial\left|\mathbf{x}-\mathbf{x}_{\mathbf{S}}\right|}=\frac{\left|\mathbf{x}-\mathbf{x}_{\mathbf{S}}\right|}{F y-r},
\end{array}\right.
$$




$$
\left\{\begin{aligned}
\frac{\partial x}{\partial \phi}=r_{2} & \left(\cos \phi+\sin \phi \frac{F x}{F y-r}\left(\cos \theta_{i}-C\right)\right. \\
& \left.+\frac{d h_{0}}{d r} \sin \phi \sin \theta_{i} \frac{x}{r}\right)^{-1} \\
\frac{\partial y}{\partial \phi}= & \frac{-F x}{F y-r}\left(\frac{\partial x}{\partial \phi}\right),
\end{aligned}\right.
$$

and:

$$
\frac{\partial h_{0}(r)}{\partial \star}=\frac{d h_{0}(r)}{d r}\left(\frac{x}{r}\left(\frac{\partial x}{\partial \star}\right)+\frac{y}{r}\left(\frac{\partial y}{\partial \star}\right)\right)
$$

so that the change of variables in Eq.(8) leads to:

$$
\overrightarrow{u_{z}} \cdot \overrightarrow{d \mathbf{x}_{\mathbf{S}}}=\left(\frac{2\left|\mathbf{x}-\mathbf{x}_{\mathbf{S}}\right|}{F A+B \cos \phi-C \sin ^{2} \phi}\right) d\left|\mathbf{x}-\mathbf{x}_{\mathbf{S}}\right| d \phi
$$

where $F, A, B$ and $C$ are defined in Eq. (11).

\section{Boundary values of integration}

Depending on the zone of calculation, the boundaries of integration in Eq. (10) differ and the integration has to be computed on a closed or an open path (an example of the two cases is represented on Fig. C.1. Tab. C.1 describes the different integration paths depending on the integration time $\tau$ and receiver position $\mathbf{x}$.

In the case of open contours, the boundaries are defined by the value of $\phi_{m}$ determined in Eq. (C.1) which corresponds to the angle of intersection of the outer edge of the structure with a sphere centered on $\mathbf{x}$ and of radius $c \tau$ :

$\phi_{m}=\arccos \left(\left(1+\frac{\left((c \tau)^{2}-z^{2}-(y-a)^{2}\right)\left((c \tau)^{2}-z^{2}-(y+a)^{2}\right)}{\cos ^{2} \theta_{i}\left((c \tau)^{2}-z^{2}-y^{2}-a^{2}+2 y r_{i}+2 r\left(h_{0}\left(r_{i}\right)\right) \tan \theta_{i}\right)^{2}}\right)^{-1 / 2}\right)$.

In the case of an observation point located in the zone I, the intersection start by impact point $\mathbf{x}_{\mathbf{i}}$ when $c \tau=\left|\mathbf{x}-\mathbf{x}_{\mathbf{i}}\right|$ then the contour is closed (integration over $\phi=[0: 2 \pi]$ ) as presented in Fig. C.1 (up) until $c \tau=\left|\mathbf{x}-\mathbf{x}_{\mathbf{h}}\right|$ then the integration path is open (integration over $\phi=\left[\phi_{m}: 2 \pi-\phi_{m}\right]$ ) as presented in Fig. C.1 (down) until $c \tau=\left|\mathbf{x}-\mathbf{x}_{\mathbf{b}}\right|$. 

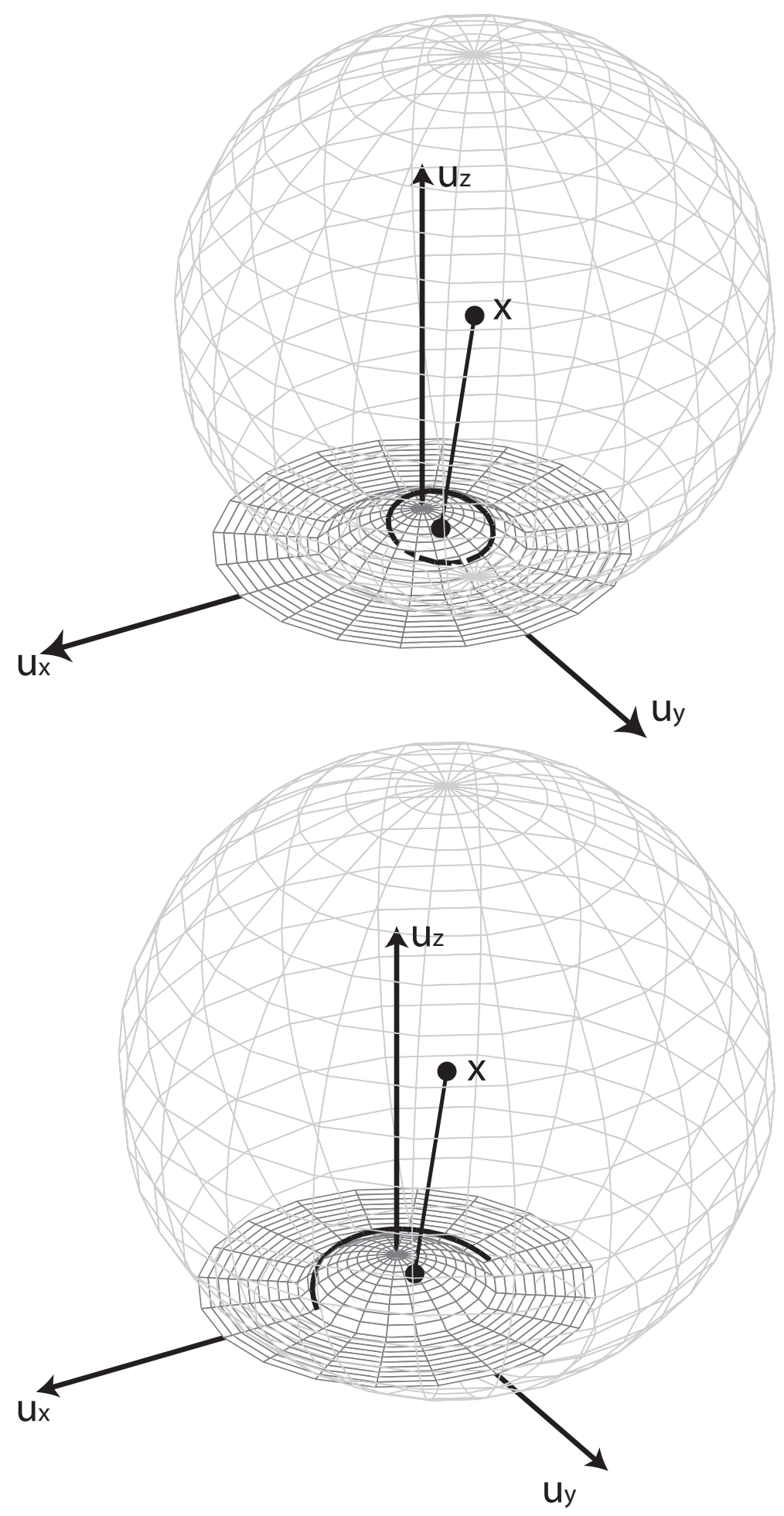

Fig. C.1. Example of a closed contour (left) and an open contour (right) of integration in the case of a spherical cap and a receiver $\mathbf{x}$ located in the zone I. The integration path corresponds to the intersection of the structure with a sphere of radius $c \tau$ centered on $\mathbf{x}$. 


\begin{tabular}{|c||c|c|c|}
\hline zone & Conditions on $\mathbf{x}$ and $\tau$ & Contour & Integration over $\phi$ \\
\hline \hline I & $\left|\mathbf{x}-\mathbf{x}_{\mathbf{i}}\right|<c \tau<\left|\mathbf{x}-\mathbf{x}_{\mathbf{h}}\right|$ & Closed & {$[0: 2 \pi]$} \\
& $\left|\mathbf{x}-\mathbf{x}_{\mathbf{h}}\right|<c \tau<\left|\mathbf{x}-\mathbf{x}_{\mathbf{b}}\right|$ & Open & {$\left[\phi_{m}: 2 \pi-\phi_{m}\right]$} \\
\hline II & $\left|\mathbf{x}-\mathbf{x}_{\mathbf{h}}\right|<c \tau<\left|\mathbf{x}-\mathbf{x}_{\mathbf{b}}\right|$ & Open & {$\left[\phi_{m}: 2 \pi-\phi_{m}\right]$} \\
\hline III & $\left|\mathbf{x}-\mathbf{x}_{\mathbf{h}}\right|<c \tau<\left|\mathbf{x}-\mathbf{x}_{\mathbf{b}}\right|$ & Open & {$\left[-\phi_{m}: \phi_{m}\right]$} \\
& $\left|\mathbf{x}-\mathbf{x}_{\mathbf{b}}\right|<c \tau<\left|\mathbf{x}-\mathbf{x}_{\mathbf{i}}\right|$ & Closed & {$[0: 2 \pi]$} \\
\hline
\end{tabular}

Table C.1

Definition of the integration contours depending on the zone, receiver location $\mathbf{x}$ and integration time $\tau=\left|\mathbf{x}-\mathbf{x}_{\mathbf{S}}\right| / c$. For open contours, the boundary are determined by $\phi_{m}$ defined in Eq. (C.1).

\section{References}

[1] W. Klippel, Nonlinear large signal behavior of electrodynamic loudspeakers at low frequencies. Journal of the Audio Engineering Society (1992) 40(6), 483496.

[2] N. Quaegebeur and A. Chaigne, Mechanical resonances and geometrical nonlinearities in electrodynamic loudspeakers. Journal of the Audio Engineering Society (2008) 56(8), 462-473.

[3] S. Sridhar, D.T. Mook and A.H. Nayfeh, Nonlinear resonances in the forced responses of plates. Part 1: symetric responses of circular plates. Journal of Sound and Vibration (1975) 41, 359-373.

[4] C. Touzé, C. Camier, G. Favraud, and O. Thomas, Effect of Imperfections and Damping on the Type of Nonlinearity of Circular Plates and Shallow Spherical Shells. Mathematical Problems in Engineering (2008) Article ID 678307, 19 pages.

[5] N. Quaegebeur and A. Chaigne, Nonlinear vibrations of loudspeaker-like structures. Journal of Sound and Vibration (2008) 309(1-2), 178-196.

[6] G.R. Harris, Review of transient field theory for a baffled planar piston. Journal of the Acoustical Society of America (1981) 70(1), 10-19.

[7] P.R. Stepanishen, Transient radiation from pistons in an infinite planar baffle. Journal of the Acoustical Society of America (1971) 49(5), 1629-1638.

[8] J.A. Jensen, A new calculation procedure for Spatial Impulse Responses in ultrasound. Journal of the Acoustical Society of America (1999) 105(1), 32663274 .

[9] G.R. Harris, Transient field of a baffled planar piston having an arbitrary vibration amplitude. Journal of the Acoustical Society of America (1981) 70(1), 186-204. 
[10] Q. Hu and S.F. Wu, An explicit integral formulation for transient acoustic radiation. Journal of the Acoustical Society of America (1998) 104(6), 32513258 .

[11] S.F. Wu and Q. Hu, An alternative formulation for predicting sound radiation from a vibrating object. Journal of the Acoustical Society of America (1998) 103(4), 1763-1774.

[12] H.T. O'Neil, Theory of focusing radiators. Journal of the Acoustical Society of America (1949) 21(5), 516-526.

[13] M. Arditi, F.S. Foster and J.W. Hunt, Transient fields of concave annular arrays. Ultrasonic Imaging (1981) 3(1), 37-61.

[14] J. M. Kates, Radiation from a Dome. Journal of the Audio Engineering Society (1976) 24(9), 735-737.

[15] W.A. Verhoef, M.J.T.M. Cloostermans and J.M. Thijssen, The impulse response of a focused source with an arbitrary axisymmetric surface velocity distribution. Journal of the Acoustical Society of America (1984) 75(6), 17161721.

[16] J.A. Ketterling, Acoustic field of a wedge-shaped section of a spherical cap transducer. Journal of the Acoustical Society of America (2003) 114(6), 30653075 .

[17] C.L.S. Farn and H. Huang, Transient acoustic fields generated by a body of arbitrary shape. Journal of the Acoustical Society of America (1968) 43(2), 252-257.

[18] D. Guyomar and J. Powers, Transient radiation from axially symmetric sources. Journal of the Acoustical Society of America (1986) 79(2), 273-277.

[19] P.R. Stepanishen, Acoustic axisymmetric radiation and scattering from bodies of revolution using the internal source density and Fourier methods. Journal of the Acoustical Society of America (1997) 102(2), 726-732.

[20] D.J. Chappell, P.J. Harris, D. Henwood and R. Chakrabarti, A stable boundary element method for modeling transient acoustic radiation. Journal of the Acoustical Society of America (2006) 120(1), 74-80.

[21] M. Bluck and S.P. Walker, Analysis of three-dimensional transient acoustic wave propagation unsing the boundary integral equation method. International Journal for Numerical Methods in Engineering (1996) 39(9), 1419-1431.

[22] H. Suzuki and J. Tichy, Sound radiation from convex and concave domes in an infinite baffle. Journal of the Acoustical Society of America (1981) 69(1), 41-49.

[23] H. Suzuki and J. Tichy, Radiation and Diffraction Effects by Convex and Concave Dome. Journal of the Audio Engineering Society (1981) 29(12), 873881. 
[24] M.C. Junger and D. Feit, Sound, structures and their interaction (1972) MIT, Cambridge.

[25] G. Lemarquand, New Structure of Loudspeaker. 120th AES Convention, Paris (2006) Paper n6846.

[26] W. Kaplan, Advanced Calculus, 4th ed. (1992) Addison-Wesley.

[27] R. Bracewell, Convolution Theorem : The Fourier Transform and Its Applications, 3rd ed. (1999) McGraw-Hill. 